\title{
A Decomposition Method for Solving the Fractional Davey-Stewartson Equations
}

\author{
H. Jafari • H. Tajadodi • \\ A. Bolandtalat · S. J. Johnston
}

Published online: 1 February 2015

(C) Springer India Pvt. Ltd. 2015

\begin{abstract}
In the present paper, we apply the new iterative method which proposed by Daftardar-Gejji and Jafari to solve fractional Davey-Stewartson equations. The convergence of this method is proved. The results obtained by this method have been compared with the exact solutions and show that proposed method is accuracy and convenience for solving nonlinear fractional differential equations.
\end{abstract}

Keywords Iterative method - Fractional Davey-Stewartson equation - Caputo fractional derivative

\section{Introduction}

Fractional calculus and fractional dynamics become hot topics of research which rapid development and implementations in various fields of engineering and science $[3,8,15]$. As a result the fractional differential equations (FDEs) started to be used in describing of real world phenomena $[3,8]$. The analytic results on uniqueness and existence of solutions of FDE were favorite topics of many researches $[3,15]$. For most of the FDEs, obtaining the analytical solutions is not easy, thus, the approximation and numerical techniques are utilized to solve them. Some of these methods were Adomian decomposition method (ADM) [1,14,16-19], Homotopy perturbation method (HPM) [1,13], Homotopy analysis method (HAM) [11],

H. Jafari $(\varangle) \cdot$ S. J. Johnston

Department of Mathematical Sciences, University of South Africa (UNISA), PO Box 392,

UNISA 0003, South Africa

e-mail: jafari@umz.ac.ir; jafarh@unisa.ac.za

H. Jafari

Department of Mathematics, University of Mazandaran, Babolsar, Iran

H. Tajadodi

Department of Mathematics, University of Sistan and Baluchestan, Zahedan, Iran

A. Bolandtalat

Department of Mathematics, Science and Research Branch, Islamic Azad University, Tehran, Iran 
Variational iteration method (VIM) [10] and so on [2]. Our main aim in the current paper is to apply new iterative method to solve fractional Davey-Stewartson (DS) equations. This method is called Daftardar-Jafari method (DJM) by Daftardar and Jafari [6].

The core of this approach is to solve nonlinear equations without using Adomian polynomials [7]. In [4] Bhalekar and Daftardar-Gejji showed the method is convergent.

The present paper,we focus on obtaining the numerical solution of the following fractional Davey-Stewartson (DS) equations:

$$
\begin{aligned}
& \frac{1}{2} \sigma^{4} \frac{\partial^{\alpha} q}{\partial y^{\alpha}}+\frac{1}{2} \sigma^{2} \frac{\partial^{2} q}{\partial x^{2}}+i \frac{\partial q}{\partial t}+\lambda|q|^{2} q-\frac{\partial \phi}{\partial x} q=0, \\
& \frac{\partial^{2} \phi}{\partial x^{2}}-\sigma^{2} \frac{\partial^{\alpha} \phi}{\partial y^{\alpha}}-2 \lambda \frac{\partial\left(|q|^{2}\right)}{\partial x}=0, \quad 1<\alpha \leq 2
\end{aligned}
$$

where $\frac{\partial^{\alpha}}{\partial y^{\alpha}}$ denotes the Caputo derivative, namely [3,15]:

$$
D_{y}^{\alpha} f(x, y, t)=\frac{\partial^{\alpha} f(x, y, t)}{\partial y^{\alpha}}= \begin{cases}I^{m-\alpha}\left[\frac{\partial^{m} f(x, y, t)}{\partial y^{m}}\right], & m-1<\alpha \leq m, \\ \frac{\partial^{m} f(x, y, t)}{\partial y^{m}}, & \alpha=m \in N .\end{cases}
$$

$I^{\alpha}$ denotes the Riemann-Liouville fractional integral, namely $[3,15]$ :

$$
\begin{aligned}
I_{y}^{\alpha} f(x, y, t) & =\frac{1}{\Gamma(\alpha)} \int_{0}^{y}(y-\tau)^{\alpha-1} f(x, \tau, t) d \tau, \quad \alpha>0, \quad y>0 \\
I_{y}^{0} f(x, y, t) & =f(x, y, t)
\end{aligned}
$$

Below the reader can find some its basic properties:

$$
\begin{aligned}
& \text { 1) } D_{y}^{\alpha} I_{y}^{\alpha} f(x, y, t)=f(x, y, t) \\
& \text { 2) } I_{y}^{\alpha} D_{y}^{\alpha} f(x, y, t)=f(x, y, t)-\sum_{k=0}^{m-1} f^{(k)}\left(x, 0^{+}, t\right) \frac{y^{k}}{k !}, \quad y>0
\end{aligned}
$$

In Eq. (1) we have the DS-I equation in the case $\sigma=1$, while $\sigma=i$ is the DS-II equation. The parameter $\lambda$ illustrates the focusing or defocusing case. The known examples of integrable equations are the Davey-Stewarston I and II. They apply in gravity-capillarity surface wave packets in the limit of the shallow water. Jafari et al. applied VIM to solve the classical and fractional Davey-Stewartson equations $[5,9,10]$.

Now we are ready to present the organization of our wok: In "Daftardar-Jafari method" section, Daftardar-Jafari method is presented. We apply the proposed technique to solve the fractional Davey-Stewartson equation in the next section. A brief conclusion is shown in "Conclusion" section.

\section{Daftardar-Jafari Method}

In this section, we now construct the iterative method by using the following decomposition method, which is mainly due to Daftardar-Gejji and Jafari [6]. This decomposition of the nonlinear function is quite different from that of Adomain decomposition. we describe the 
application of Daftardar-Jafari method to solve the fractional Davey-Stewartson equations:

$$
\begin{aligned}
& \frac{1}{2} \sigma^{4} \frac{\partial^{\alpha} q}{\partial y^{\alpha}}+\frac{1}{2} \sigma^{2} \frac{\partial^{2} q}{\partial x^{2}}+i \frac{\partial q}{\partial t}+\lambda|q|^{2} q-\frac{\partial \phi}{\partial x} q=0, \\
& \frac{\partial^{2} \phi}{\partial x^{2}}-\sigma^{2} \frac{\partial^{\alpha} \phi}{\partial y^{\alpha}}-2 \lambda \frac{\partial\left(|q|^{2}\right)}{\partial x}=0, \quad 1<\alpha \leq 2 .
\end{aligned}
$$

In systeam (5) we divide into real part and imaginary part the amplitude of a surface wave packet $q$ and rewrite (5) in the following form:

$$
\begin{aligned}
& \frac{\partial^{\alpha} u}{\partial y^{\alpha}}+\frac{1}{\sigma^{2}} \frac{\partial^{2} u}{\partial x^{2}}-\frac{2}{\sigma^{4}} \frac{\partial v}{\partial t}+\frac{2 \lambda}{\sigma^{4}}\left(u^{3}+v^{2} u\right)-\frac{2}{\sigma^{4}}\left(\frac{\partial \phi}{\partial x} u\right)=0, \\
& \frac{\partial^{\alpha} v}{\partial y^{\alpha}}+\frac{1}{\sigma^{2}} \frac{\partial^{2} v}{\partial x^{2}}+\frac{2}{\sigma^{4}} \frac{\partial u}{\partial t}+\frac{2 \lambda}{\sigma^{4}}\left(v^{3}+u^{2} v\right)-\frac{2}{\sigma^{4}}\left(\frac{\partial \phi}{\partial x} v\right)=0, \\
& \frac{\partial^{\alpha} \phi}{\partial y^{\alpha}}-\frac{1}{\sigma^{2}} \frac{\partial^{2} \phi}{\partial x^{2}}+\frac{2 \lambda}{\sigma^{2}} \frac{\partial\left(u^{2}+v^{2}\right)}{\partial x}=0 .
\end{aligned}
$$

In view of Eq. (4), applying $I^{\alpha}$ on both sides of Eq. (6), we get the following integral equations:

$$
\begin{aligned}
u(x, y, t)= & u(x, 0, t)+\sum_{k=0}^{m-1} u^{(k)}(x, 0, t) \frac{y^{k}}{k !}-I^{\alpha}\left[\frac{1}{\sigma^{2}} \frac{\partial^{2} u}{\partial x^{2}}-\frac{2}{\sigma^{4}} \frac{\partial v}{\partial t}+\frac{2 \lambda}{\sigma^{4}}\left(u^{3}+v^{2} u\right)\right. \\
& \left.-\frac{2}{\sigma^{4}}\left(\frac{\partial \phi}{\partial x} u\right)\right]=f_{1}+N_{1}(u, v, \phi), \\
v(x, y, t)= & v(x, 0, t)+\sum_{k=0}^{m-1} v^{(k)}(x, 0, t) \frac{y^{k}}{k !}-I^{\alpha}\left[\frac{1}{\sigma^{2}} \frac{\partial^{2} v}{\partial x^{2}}+\frac{2}{\sigma^{4}} \frac{\partial u}{\partial t}+\frac{2 \lambda}{\sigma^{4}}\left(v^{3}+u^{2} v\right)\right. \\
& \left.-\frac{2}{\sigma^{4}}\left(\frac{\partial \phi}{\partial x} v\right)\right]=f_{2}+N_{2}(u, v, \phi), \\
\phi(x, y, t)= & \phi(x, 0, t)+\sum_{k=0}^{m-1} \phi^{(k)}(x, 0, t) \frac{y^{k}}{k !}-I^{\alpha}\left[-\frac{1}{\sigma^{2}} \frac{\partial^{2} \phi}{\partial x^{2}}+\frac{2 \lambda}{\sigma^{2}} \frac{\partial\left(u^{2}+v^{2}\right)}{\partial x}\right] \\
= & f_{3}+N_{3}(u, v, \phi) .
\end{aligned}
$$

where

$$
\begin{aligned}
f_{1} & =u(x, 0, t)+\sum_{k=0}^{m-1} u^{(k)}(x, 0, t) \frac{y^{k}}{k !} \\
N_{1}(u, v, \phi) & =-I^{\alpha}\left[\frac{1}{\sigma^{2}} \frac{\partial^{2} u}{\partial x^{2}}-\frac{2}{\sigma^{4}} \frac{\partial v}{\partial t}+\frac{2 \lambda}{\sigma^{4}}\left(u^{3}+v^{2} u\right)-\frac{2}{\sigma^{4}}\left(\frac{\partial \phi}{\partial x} u\right)\right], \\
f_{2} & =v(x, 0, t)+\sum_{k=0}^{m-1} v^{(k)}(x, 0, t) \frac{y^{k}}{k !}, \\
N_{2}(u, v, \phi) & =-I^{\alpha}\left[\frac{1}{\sigma^{2}} \frac{\partial^{2} v}{\partial x^{2}}+\frac{2}{\sigma^{4}} \frac{\partial u}{\partial t}+\frac{2 \lambda}{\sigma^{4}}\left(v^{3}+u^{2} v\right)-\frac{2}{\sigma^{4}}\left(\frac{\partial \phi}{\partial x} v\right)\right],
\end{aligned}
$$




$$
\begin{gathered}
f_{3}=\phi(x, 0, t)+\sum_{k=0}^{m-1} \phi^{(k)}(x, 0, t) \frac{y^{k}}{k !}, \\
N_{3}(u, v, \phi)=-I^{\alpha}\left[-\frac{1}{\sigma^{2}} \frac{\partial^{2} \phi}{\partial x^{2}}+\frac{2 \lambda}{\sigma^{2}} \frac{\partial\left(u^{2}+v^{2}\right)}{\partial x}\right],
\end{gathered}
$$

where $f_{k}(k=1,2,3)$ are known functions and $N_{k}$ are nonlinear operators. The unknown functions can be shown in terms of an infinite series in iterative decomposition method as follow:

$$
u=\sum_{i=0}^{\infty} u_{i}, \quad v=\sum_{i=0}^{\infty} v_{i}, \quad \phi=\sum_{i=0}^{\infty} \phi_{i},
$$

and the nonlinear function $N_{k},(k=1,2,3)$ can be decomposed as

$$
\begin{aligned}
& N_{1}\left(\sum_{i=0}^{\infty} u_{i}, \sum_{i=0}^{\infty} v_{i}, \sum_{i=0}^{\infty} \phi_{i}\right)=N_{1}\left(u_{0}, v_{0}, \phi_{0}\right) \\
& +\sum_{i=0}^{\infty}\left\{N_{1}\left(\sum_{j=0}^{i} u_{j}, \sum_{j=0}^{i} v_{j}, \sum_{j=0}^{i} \phi_{j}\right)\right. \\
& \left.-N_{1}\left(\sum_{j=0}^{i-1} u_{j}, \sum_{j=0}^{i-1} v_{j}, \sum_{j=0}^{i-1} \phi_{j}\right)\right\} \text {, } \\
& N_{2}\left(\sum_{i=0}^{\infty} u_{i}, \sum_{i=0}^{\infty} v_{i}, \sum_{i=0}^{\infty} \phi_{i}\right)=N_{2}\left(u_{0}, v_{0}, \phi_{0}\right) \\
& +\sum_{i=0}^{\infty}\left\{N_{2}\left(\sum_{j=0}^{i} u_{j}, \sum_{j=0}^{i} v_{j}, \sum_{j=0}^{i} \phi_{j}\right)\right. \\
& \left.-N_{2}\left(\sum_{j=0}^{i-1} u_{j}, \sum_{j=0}^{i-1} v_{j}, \sum_{j=0}^{i-1} \phi_{j}\right)\right\} \text {, } \\
& N_{3}\left(\sum_{i=0}^{\infty} u_{i}, \sum_{i=0}^{\infty} v_{i}, \sum_{i=0}^{\infty} \phi_{i}\right)=N_{3}\left(u_{0}, v_{0}, \phi_{0}\right) \\
& +\sum_{i=0}^{\infty}\left\{N_{3}\left(\sum_{j=0}^{i} u_{j}, \sum_{j=0}^{i} v_{j}, \sum_{j=0}^{i} \phi_{j}\right)\right. \\
& \left.-N_{3}\left(\sum_{j=0}^{i-1} u_{j}, \sum_{j=0}^{i-1} v_{j}, \sum_{j=0}^{i-1} \phi_{j}\right)\right\} \text {. }
\end{aligned}
$$


Substituting Eqs. (8) and (9) in Eq. (7), we have

$$
\begin{aligned}
\sum_{i=0}^{\infty} u_{i}= & f_{1}+N_{1}\left(u_{0}, v_{0}, \phi_{0}\right)+\sum_{i=0}^{\infty}\left\{N_{1}\left(\sum_{j=0}^{i} u_{j}, \sum_{j=0}^{i} v_{j}, \sum_{j=0}^{i} \phi_{j}\right)\right. \\
& \left.-N_{1}\left(\sum_{j=0}^{i-1} u_{j}, \sum_{j=0}^{i-1} v_{j}, \sum_{j=0}^{i-1} \phi_{j}\right)\right\}, \\
\sum_{i=0}^{\infty} v_{i}= & f_{2}+N_{2}\left(u_{0}, v_{0}, \phi_{0}\right)+\sum_{i=0}^{\infty}\left\{N_{2}\left(\sum_{j=0}^{i} u_{j}, \sum_{j=0}^{i} v_{j}, \sum_{j=0}^{i} \phi_{j}\right)\right. \\
& \left.-N_{2}\left(\sum_{j=0}^{i-1} u_{j}, \sum_{j=0}^{i-1} v_{j}, \sum_{j=0}^{i-1} \phi_{j}\right)\right\}, \\
\sum_{i=0}^{\infty} \phi_{i}= & f_{3}+N_{3}\left(u_{0}, v_{0}, \phi_{0}\right)+\sum_{i=0}^{\infty}\left\{N_{3}\left(\sum_{j=0}^{i} u_{j}, \sum_{j=0}^{i} v_{j}, \sum_{j=0}^{i} \phi_{j}\right)\right. \\
& \left.-N_{3}\left(\sum_{j=0}^{i-1} u_{j}, \sum_{j=0}^{i-1} v_{j}, \sum_{j=0}^{i-1} \phi_{j}\right)\right\}
\end{aligned}
$$

then we have the following recurrence relations:

$$
\begin{aligned}
& \left\{\begin{array}{l}
u_{0}=f_{1} \\
u_{1}=N_{1}\left(u_{0}, v_{0}, \phi_{0}\right) \\
u_{m+1}=N_{1}\left(u_{0}+\cdots+u_{m}, v_{0}+\cdots+v_{m}, \phi_{0}+\cdots+\phi_{m}\right) \\
\quad-N_{1}\left(u_{0}+\cdots+u_{m-1}, v_{0}+\cdots+v_{m-1}, \phi_{0}+\cdots+\phi_{m-1}\right), \quad m=1,2, \ldots
\end{array}\right. \\
& \left\{\begin{array}{l}
v_{0}=f_{2} \\
v_{1}=N_{2}\left(u_{0}, v_{0}, \phi_{0}\right) \\
v_{m+1}=N_{2}\left(u_{0}+\cdots+u_{m}, v_{0}+\cdots+v_{m}, \phi_{0}+\cdots+\phi_{m}\right) \\
\quad-N_{2}\left(u_{0}+\cdots+u_{m-1}, v_{0}+\cdots+v_{m-1}, \phi_{0}+\cdots+\phi_{m-1}\right), \quad m=1,2, \ldots
\end{array}\right. \\
& \left\{\begin{array}{l}
\phi_{0}=f_{3} \\
\phi_{1}=N_{3}\left(u_{0}, v_{0}, \phi_{0}\right) \\
\phi_{m+1}=N_{3}\left(u_{0}+\cdots+u_{m}, v_{0}+\cdots+v_{m}, \phi_{0}+\cdots+\phi_{m}\right) \\
\quad-N_{3}\left(u_{0}+\cdots+u_{m-1}, v_{0}+\cdots+v_{m-1}, \phi_{0}+\cdots+\phi_{m}-\cdots\right.
\end{array}\right. \\
& -N_{3}\left(u_{0}+\cdots+u_{m-1}, v_{0}+\cdots+v_{m-1}, \phi_{0}+\cdots+\phi_{m-1}\right), \quad m=1,2, \ldots
\end{aligned}
$$

Then

$$
\begin{aligned}
& \left(u_{1}+\cdots+u_{m+1}\right)=N_{1}\left(u_{0}+\cdots+u_{m}, v_{0}+\cdots+v_{m}, \phi_{0}+\cdots+\phi_{m}\right), \\
& \left(v_{1}+\cdots+v_{m+1}\right)=N_{2}\left(u_{0}+\cdots+u_{m}, v_{0}+\cdots+v_{m}, \phi_{0}+\cdots+\phi_{m}\right), \\
& \left(\phi_{1}+\cdots+\phi_{m+1}\right)=N_{3}\left(u_{0}+\cdots+u_{m}, v_{0}+\cdots+v_{m}, \phi_{0}+\cdots+\phi_{m}\right),
\end{aligned}
$$

and

$$
u=f_{1}+\sum_{i=1}^{\infty} u_{i}, \quad v=f_{2}+\sum_{i=1}^{\infty} v_{i}, \quad \phi=f_{3}+\sum_{i=1}^{\infty} \phi_{i}
$$


are calculated.

\section{Convergence of DJM Solutions}

In this section, we provide sufficient condition for the convergence of DJM solution series (8).

Theorem 1 If $N_{i}$ is a contraction, i.e. $\left\|N_{i}(\phi)-N_{i}(\psi)\right\| \leq k\|\phi-\psi\|, 0<k<1$ then

$$
\begin{aligned}
\left\|u_{m+1}\right\| & =\left\|N_{1}\left(\sum_{j=0}^{m} u_{j}, \sum_{j=0}^{m} v_{j}, \sum_{j=0}^{m} \phi_{j}\right)-N_{1}\left(\sum_{j=0}^{m-1} u_{j}, \sum_{j=0}^{m-1} v_{j}, \sum_{j=0}^{m-1} \phi_{j}\right)\right\| \\
& \leq k\left\|u_{m}\right\| \leq k^{m}\left\|u_{0}\right\|, \quad m=0,1,2, \ldots \\
\left\|v_{m+1}\right\| & =\left\|N_{2}\left(\sum_{j=0}^{m} u_{j}, \sum_{j=0}^{m} v_{j}, \sum_{j=0}^{m} \phi_{j}\right)-N_{2}\left(\sum_{j=0}^{m-1} u_{j}, \sum_{j=0}^{m-1} v_{j}, \sum_{j=0}^{m-1} \phi_{j}\right)\right\| \\
& \leq k\left\|v_{m}\right\| \leq k^{m}\left\|v_{0}\right\|, \quad m=0,1,2, \ldots \\
\left\|\phi_{m+1}\right\| & =\left\|N_{3}\left(\sum_{j=0}^{m} u_{j}, \sum_{j=0}^{m} v_{j}, \sum_{j=0}^{m} \phi_{j}\right)-N_{3}\left(\sum_{j=0}^{m-1} u_{j}, \sum_{j=0}^{m-1} v_{j}, \sum_{j=0}^{m-1} \phi_{j}\right)\right\| \\
& \leq k\left\|\phi_{m}\right\| \leq k^{m}\left\|\phi_{0}\right\|, \quad m=0,1,2, \ldots
\end{aligned}
$$

and the series $\sum_{i=0}^{m} u_{i}, \sum_{i=0}^{m} v_{i}$ and $\sum_{i=0}^{m} \phi_{i}$ absolutely and uniformly converges to a solution of Eq. (6), which is unique, in view of the Banach fixed point theorem [12].

Theorem 2 If the series solution defined in (15) is convergent, then it converges to an exact solution of Eq. (6).

Proof The truncated series $\sum_{i=0}^{m} u_{i}, \sum_{i=0}^{m} v_{i}$ and $\sum_{i=0}^{m} \phi_{i}$ are used an approximation to the solution $u(t), v(t)$ and $\phi(t)$ of Eq. (6), using the above we have:

$$
\begin{aligned}
\sum_{i=0}^{m} u_{i} & =\left(f+N_{1}\left(\sum_{i=0}^{m} u_{i}, \sum_{i=0}^{m} v_{i}, \sum_{i=0}^{m} \phi_{i}\right)\right) \\
\sum_{i=0}^{m} v_{i} & =\left(f+N_{1}\left(\sum_{i=0}^{m} u_{i}, \sum_{i=0}^{m} v_{i}, \sum_{i=0}^{m} \phi_{i}\right)\right) \\
\sum_{i=0}^{m} \phi_{i} & =\left(f+N_{1}\left(\sum_{i=0}^{m} u_{i}, \sum_{i=0}^{m} v_{i}, \sum_{i=0}^{m} \phi_{i}\right)\right)
\end{aligned}
$$

Taking limits of above equation, it follows that:

$$
\begin{aligned}
u=\lim _{m \rightarrow \infty} \sum_{i=0}^{m} u_{i} & =\lim _{m \rightarrow \infty}\left(f+N_{1}\left(\sum_{i=0}^{m} u_{i}, \sum_{i=0}^{m} v_{i}, \sum_{i=0}^{m} \phi_{i}\right)\right) \\
& \left.=\lim _{m \rightarrow \infty} f+\lim _{m \rightarrow \infty} N_{1}\left(\sum_{i=0}^{m} u_{i}, \sum_{i=0}^{m} v_{i}, \sum_{i=0}^{m} \phi_{i}\right)\right)
\end{aligned}
$$




$$
\begin{aligned}
& \left.=f+N_{1}\left(\lim _{m \rightarrow \infty} \sum_{i=0}^{m} u_{i}, \lim _{m \rightarrow \infty} \sum_{i=0}^{m} v_{i}, \lim _{m \rightarrow \infty} \sum_{i=0}^{m} \phi_{i}\right)\right) \\
& =f+N_{1}(u, v, \phi) \\
v=\lim _{m \rightarrow \infty} \sum_{i=0}^{m} u_{i} & =\lim _{m \rightarrow \infty}\left(f+N_{2}\left(\sum_{i=0}^{m} u_{i}, \sum_{i=0}^{m} v_{i}, \sum_{i=0}^{m} \phi_{i}\right)\right) \\
& \left.=\lim _{m \rightarrow \infty} f+\lim _{m \rightarrow \infty} N_{2}\left(\sum_{i=0}^{m} u_{i}, \sum_{i=0}^{m} v_{i}, \sum_{i=0}^{m} \phi_{i}\right)\right) \\
& \left.=f+N_{2}\left(\lim _{m \rightarrow \infty} \sum_{i=0}^{m} u_{i}, \lim _{m \rightarrow \infty} \sum_{i=0}^{m} v_{i}, \lim _{m \rightarrow \infty} \sum_{i=0}^{m} \phi_{i}\right)\right) \\
& =f+N_{2}(u, v, \phi) \\
\lim _{m \rightarrow \infty} \sum_{i=0}^{m} u_{i} & =\lim _{m \rightarrow \infty}\left(f+N_{3}\left(\sum_{i=0}^{m} u_{i}, \sum_{i=0}^{m} v_{i}, \sum_{i=0}^{m} \phi_{i}\right)\right) \\
& \left.=\lim _{m \rightarrow \infty} f+\lim _{m \rightarrow \infty} N_{3}\left(\sum_{i=0}^{m} u_{i}, \sum_{i=0}^{m} v_{i}, \sum_{i=0}^{m} \phi_{i}\right)\right) \\
& \left.=f+N_{3}\left(\lim _{m \rightarrow \infty} \sum_{i=0}^{m} u_{i}, \lim _{m \rightarrow \infty} \sum_{i=0}^{m} v_{i}, \lim _{m \rightarrow \infty} \sum_{i=0}^{m} \phi_{i}\right)\right) \\
& =f+N_{3}(u, v, \phi)
\end{aligned}
$$

Hence $u, v$ and $\phi$ are the solutions of Eq. (6) and the proof is complete.

\section{Applications}

Below we use the presented approach in order to solve the fractional Davey-Stewartson equations (6). Subject to the following initial conditions:

$$
\begin{aligned}
u(x, 0, t) & =r \operatorname{sech}[s(x-c t)] \cos \left[\left(k_{1} x+k_{3} t\right)\right], \\
v(x, 0, t) & =r \operatorname{sech}[s(x-c t)] \sin \left[\left(k_{1} x+k_{3} t\right)\right], \\
\phi(x, 0, t) & =f \tanh [s(x-c t)],
\end{aligned}
$$

where

$$
\begin{array}{ll}
c=k_{2}+\sigma^{2} k_{1}, & r=\sqrt{-\frac{\left(2 k_{3}+k_{1}^{2} \sigma^{2}+k_{2}^{2}\right.}{\lambda},} \\
f=\frac{2 \sigma \sqrt{-\lambda}}{1-\sigma^{2}}, & s=\sqrt{\frac{2 k_{3}+k_{1}^{2} \sigma^{2}+k_{2}^{2}}{\sigma^{2}}},
\end{array}
$$

and $k_{i},(i=1,2,3)$ are arbitrary constants. The accuracy solution in the special case $\alpha=2$ [9]:

$$
\begin{aligned}
& u(x, y, t)=r \operatorname{sech}[s(x+y-c t)] \cos \left[k_{1} x+k_{2} y+k_{3} t\right], \\
& v(x, y, t)=r \operatorname{sech}[s(x+y-c t)] \sin \left[k_{1} x+k_{2} y+k_{3} t\right], \\
& \phi(x, y, t)=f \tanh [s(x+y-c t)] .
\end{aligned}
$$


According to the Daftardar-Jafari method and applying the algorithm given in (11), (12), (13) to Eq. (7), we get:

$$
\begin{aligned}
& \left\{\begin{aligned}
u_{0}= & r \operatorname{sech}[s(x-c t)] \cos \left[\left(k_{1} x+k_{3} t\right)\right]+y(-r s \operatorname{sech}[s(x-c t)] \tanh [s(x-c t)] \\
& \left.\left.\times \cos \left[k_{1} x+k_{3} t\right)\right]-r k_{2} \operatorname{sech}[s(x-c t)] \sin \left[\left(k_{1} x+k_{3} t\right)\right]\right) \\
u_{1}= & N_{1}\left(u_{0}, v_{0}, \phi_{0}\right) \\
u_{m+1}= & N_{1}\left(\sum_{j=0}^{m} u_{j}, \sum_{j=0}^{m} v_{j}, \sum_{j=0}^{m} \phi_{j}\right)-N_{1}\left(\sum_{j=0}^{m-1} u_{j}, \sum_{j=0}^{m-1} v_{j}, \sum_{j=0}^{m-1} \phi_{j}\right) m=1,2, \cdots
\end{aligned}\right. \\
& \left\{\begin{aligned}
v_{0}= & r \operatorname{sech}[s(x-c t)] \sin \left[\left(k_{1} x+k_{3} t\right)\right]+y(-r s \operatorname{sech}[s(x-c t)] \tanh [s(x-c t)] \\
& \left.\left.\times \cos \left[k_{1} x+k_{3} t\right)\right]+r k_{2} \operatorname{sech}[s(x-c t)] \sin \left[\left(k_{1} x+k_{3} t\right)\right]\right) \\
v_{1}= & N_{2}\left(u_{0}, v_{0}, \phi_{0}\right) \\
v_{m+1}= & N_{2}\left(\sum_{j=0}^{m} u_{j}, \sum_{j=0}^{m} v_{j}, \sum_{j=0}^{m} \phi_{j}\right)-N_{2}\left(\sum_{j=0}^{m-1} u_{j}, \sum_{j=0}^{m-1} v_{j}, \sum_{j=0}^{m-1} \phi_{j}\right) m=1,2, \cdots \\
\phi_{1}= & N_{3}\left(u_{0}, v_{0}, \phi_{0}\right) \\
\phi_{m+1}= & N_{3}\left(\sum_{j=0}^{m} u_{j}, \sum_{j=0}^{m} v_{j}, \sum_{j=0}^{m} \phi_{j}\right)-N_{3}\left(\sum_{j=0}^{m-1} u_{j}, \sum_{j=0}^{m-1} v_{j}, \sum_{j=0}^{m-1} \phi_{j}\right) m=1,2, \cdots
\end{aligned}\right.
\end{aligned}
$$

Tables 1,2, and 3 show the absolute errors between the exact solution together with the approximation solutions obtained for value of $\alpha=1.98$ by the DJM. The approximate solutions for $\alpha=1.98, \alpha=1.8$ and the exact solutions are plotted in Figs. 1,2, and 3.We used the two-order term of the DJM solutions for the special case $y=0.2, k_{1}=0.1, k_{2}=$ $0.03, k_{3}=-0.3, \sigma=i, \lambda=1$ for Tables 1,2, and 3 and Figs. 1,2, and 3 .

Table 1 Absolute errors of $u(x, y, t)$

\begin{tabular}{llllll}
\hline$x / t$ & 0.1 & 0.2 & 0.3 & 0.4 & 0.5 \\
\hline 20 & $1.77764 \times 10^{-9}$ & $1.08873 \times 10^{-9}$ & $9.99789 \times 10^{-9}$ & $9.10885 \times 10^{-9}$ & $8.22106 \times 10^{-9}$ \\
17 & $3.25493 \times 10^{-9}$ & $2.31386 \times 10^{-9}$ & $1.38087 \times 10^{-9}$ & $4.56749 \times 10^{-10}$ & $4.57754 \times 10^{-10}$ \\
14 & $6.26074 \times 10^{-8}$ & $7.16917 \times 10^{-8}$ & $8.0611 \times 10^{-8}$ & $8.93589 \times 10^{-8}$ & $9.7929 \times 10^{-8}$ \\
11 & $1.59523 \times 10^{-6}$ & $1.67397 \times 10^{-6}$ & $1.7503 \times 10^{-6}$ & $1.82419 \times 10^{-6}$ & $1.89559 \times 10^{-6}$ \\
7 & $5.94554 \times 10^{-5}$ & $6.05265 \times 10^{-5}$ & $6.15300 \times 10^{-5}$ & $6.24567 \times 10^{-5}$ & $6.33335 \times 10^{-5}$ \\
\hline
\end{tabular}

Table 2 Absolute errors of $v(x, y, t)$

\begin{tabular}{llllll}
\hline$x / t$ & 0.1 & 0.2 & 0.3 & \multicolumn{1}{l}{0.4} & \multicolumn{1}{l}{0.5} \\
\hline 20 & $2.74744 \times 10^{-9}$ & $2.76637 \times 10^{-9}$ & $2.78254 \times 10^{-9}$ & $2.79596 \times 10^{-9}$ & $2.80665 \times 10^{-9}$ \\
17 & $3.09023 \times 10^{-8}$ & $3.08173 \times 10^{-8}$ & $3.07046 \times 10^{-8}$ & $3.05648 \times 10^{-8}$ & $3.03982 \times 10^{-8}$ \\
14 & $3.16888 \times 10^{-7}$ & $3.13152 \times 10^{-7}$ & $3.09167 \times 10^{-7}$ & $3.0494 \times 10^{-7}$ & $3.00476 \times 10^{-7}$ \\
11 & $2.95467 \times 10^{-6}$ & $2.88966 \times 10^{-6}$ & $2.82268 \times 10^{-6}$ & $2.75382 \times 10^{-6}$ & $2.68317 \times 10^{-6}$ \\
7 & $4.76616 \times 10^{-5}$ & $4.56068 \times 10^{-5}$ & $4.35321 \times 10^{-5}$ & $4.14398 \times 10^{-5}$ & $3.93319 \times 10^{-5}$ \\
\hline
\end{tabular}


Table 3 Absolute errors of $\phi(x, y, t)$

\begin{tabular}{llllll}
\hline$x / t$ & 0.1 & 0.2 & 0.3 & 0.4 & 0.5 \\
\hline 20 & $9.99201 \times 10^{-16}$ & $8.88178 \times 10^{-16}$ & $8.88178 \times 10^{169}$ & $9.99201 \times 10^{-16}$ & $9.99201 \times 10^{-16}$ \\
17 & $1.06359 \times 10^{-13}$ & $1.05249 \times 10^{-13}$ & $1.04028 \times 10^{-13}$ & $1.02918 \times 10^{-13}$ & $1.01696 \times 10^{-13}$ \\
14 & $1.14874 \times 10^{-11}$ & $1.13626 \times 10^{-11}$ & $1.1239 \times 10^{-11}$ & $1.11169 \times 10^{-11}$ & $1.09961 \times 10^{-11}$ \\
11 & $1.24133 \times 10^{-9}$ & $1.22784 \times 10^{-9}$ & $1.22784 \times 10^{-9}$ & $1.2145 \times 10^{-9}$ & $1.18825 \times 10^{-9}$ \\
7 & $6.38877 \times 10^{-7}$ & $6.31934 \times 10^{-7}$ & $6.25068 \times 10^{-7}$ & $6.18276 \times 10^{-7}$ & $6.11557 \times 10^{-7}$ \\
\hline
\end{tabular}
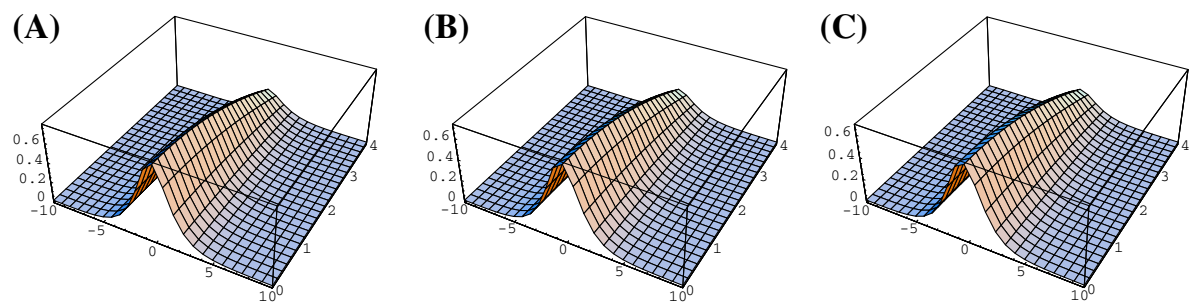

Fig. 1 The surface shows the solution $u(x ; y ; t)$ for Eq. (7): a exact solution; b approximate solution for $\alpha=1.98$; c approximate solution for $\alpha=1.8$
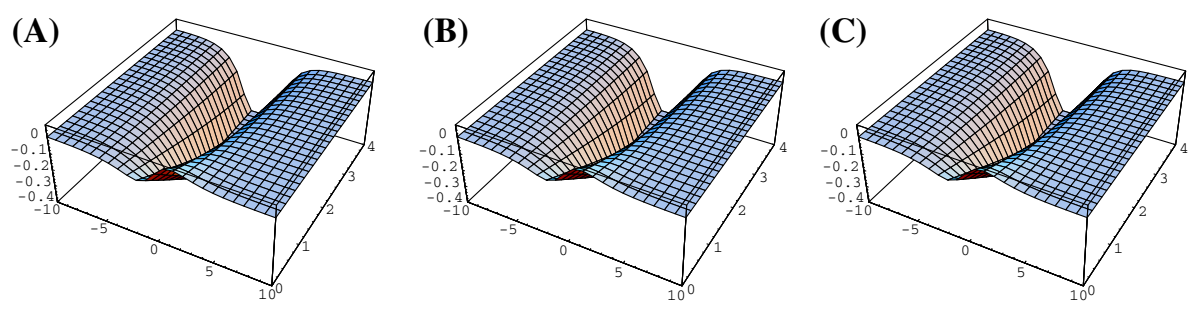

Fig. 2 The surface shows the solution $v(x ; y ; t)$ for Eq. (7): a exact solution; b approximate solution for $\alpha=1.98$; c approximate solution for $\alpha=1.8$
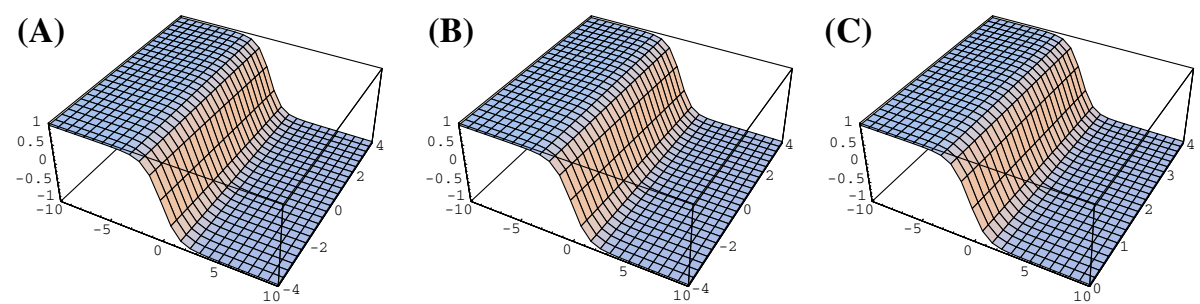

Fig. 3 The surface shows the solution $\phi(x ; y ; t)$ for Eq. (7): a exact solution; b approximate solution for $\alpha=1.98 ; \mathbf{c}$ approximate solution for $\alpha=1.8$

\section{Conclusion}

In this work, we applied the Daftardar-Jafari method to solve fractional Davey-Stewartson differential equations. The results show that this method is accurate and effective and can be used for nonlinear fractional differential equations. This method have an advantage over ADM that DJM can be solved nonlinear problems without using Adomian polynomials. 
Mathematica has been used for computations and programming in this paper.

\section{References}

1. Atangana, A., Belhaouari, S.B.: Solving partial differential equation with space-and time-fractional derivatives via homotopy decomposition method. Math. Probl. Eng. 102, 1-9 (2013)

2. Atangana, A., Vermeulen, P.D.: Analytical solutions of a space-time fractional derivative of groundwater flow equation. Abstr. Appl. Anal. 2014, 1-11 (2014)

3. Baleanu, D., Diethelm, K., Scalas, E., Trujillo, J.J.: Fractional Calculus Models and Numerical Methods Series on Complexity Nonlinearity and Chaos. World Scientific, Boston (2012)

4. Bhalekar, S., Daftardar-Gejji, V. : Convergence of the new iterative method. Int. J. Differ. Equ. (2011). doi:10.1155/2011/989065

5. Bhalekar, S., Daftardar-Gejji, V.: Solving evolution equations using a new iterative method. Numer. Methods Partial Differ. Equ. 26(4), 906-916 (2010)

6. Daftardar-Gejji, V., Jafari, H.: An iterative method for solving nonlinear functional equations. J. Math. Anal. Appl. 316, 753-763 (2006)

7. Duan, J.-S., Rach, R.: A new modification of the Adomian decomposition method for solving boundary value problems for higher order nonlinear differential equations. Appl. Math. Comput. 218(8), 4090-4118 (2011)

8. Jafari, H.: An Introduction to Fractional Differential Equations. Mazandaran University Press, Persian (2013)

9. Jafari, H., Alipour, M.: Numerical solution of Davey-Stewartson equations using variational iteration method. World Appl. Sci. J. 8, 814-819 (2010)

10. Jafari, H., Kadem, A., Baleanu, D., Yilmaz, T.: Solutions of the fractional Davey-Stewartson equations with variational iteration method. Rom. Rep. Phys. 64(2), 337-346 (2012)

11. Jafari, H., Sayevand, K., Tajadodi, H., Baleanu, D.: Homotopy analysis method for solving Abel differential equation of fractional order. Cen. Eur. J. Phys. 11(10), 1523-1527 (2013)

12. Jerri, A.J.: Introduction to Integral Equations with Applications, 2nd edn. Wiley-Interscience, New York (1999)

13. Momani, S., Odibat, Z.: Homotopy perturbation method for nonlinear partial differential equations of fractional order. Phys. Lett. A 365, 345-350 (2007)

14. Momani, S., Al-Khaled, K.: Numerical solutions for systems of fractional differential equations by the decomposition method. Appl. Math. comput. 162(3), 1351-1365 (2005)

15. Podlubny, I.: Fractional Differential Equations. Academic Press, New York (1999)

16. Saha Ray, S., Bera, R.K.: An approximate solution of a nonlinear fractional differential equation by Adomian decomposition method. Appl. Math. Comput. 167(1), 561-571 (2005)

17. Saha Ray, S., Bera, R.K.: Analytical solution of a fractional diffusion equation by Adomian decomposition method. Appl. Math. Comput. 174(1), 329-336 (2006)

18. Saha Ray, S.: A numerical solution of the coupled Sine-Gordon equation using the modified decomposition method. Appl. Math. Comput. 175(2), 1046-1054 (2006)

19. Saha Ray, S.: Exact solutions for time-fractional diffusion-wave equations by decomposition method. Physica Scripta 75(1), 53-61 (2007) 